\title{
Basic Study of Heating Response Measurement for Nanosheet Particle/Polymer Composite Gel Actuator with Anisotropic Contraction
}

\author{
Hitoshi KINO ${ }^{1, a}$, Akihiro KIYOTA ${ }^{1, b}$, Nobuyoshi MIYAMOTO ${ }^{1, c}$, \\ Takumi INADOMI ${ }^{1, \mathrm{~d}}$, Tomonori KATO ${ }^{1, \mathrm{e}}$ and Hiroyuki FUJIOKA ${ }^{1, \mathrm{f}}$
}

\author{
13-30-1, Wajiro-higashi, Higashi-ku, Fukuoka, 811-0295 Japan

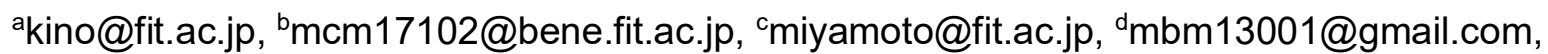 \\ et-kato@fit.ac.jp, ffujioka@fit.ac.jp
}

Keywords: control, step response, soft actuator, measurement instrument

\begin{abstract}
A soft actuator is expected to be applied in the next generation of robotics. This study focuses on soft gel actuators hybridized with nanosheet liquid crystals. The resulting soft actuator has a highly hydrophilic property, and is suitable for underwater use. When the gel in water is heated to more than the transition temperature, the gel contracts; conversely, it swells when it is cooled. This gel actuator remarkably has an anisotropic contraction characteristic because the orientation of the nanosheets is uniformly arranged. However, little is known about its dynamic characteristics of the thermal contraction. As a basic analysis, this paper investigates the heating step response through experiments, and reveals the variation of the time constant against the dimensions.
\end{abstract}

\section{Introduction}

A soft actuator has attracted attention as a part of scientific research in innovative areas [1-4]; it is expected to be applied to the next generation of robotics $[5,6]$. This study focuses on soft gel actuators hybridized with nanosheet liquid crystals, and aims to apply the results to robotics. As shown in Fig. 1 , the gel actuator is made of poly $\mathrm{N}$-isopropylacrylamide hydrogel, which consists of nanosheet liquid crystals (fluoro-hectorite), methylene-bisacrylamide, and water. Because the contained water approximately accounts for $90 \%$ of the gel, the gel actuator has a highly hydrophilic property and is suitable for underwater use.
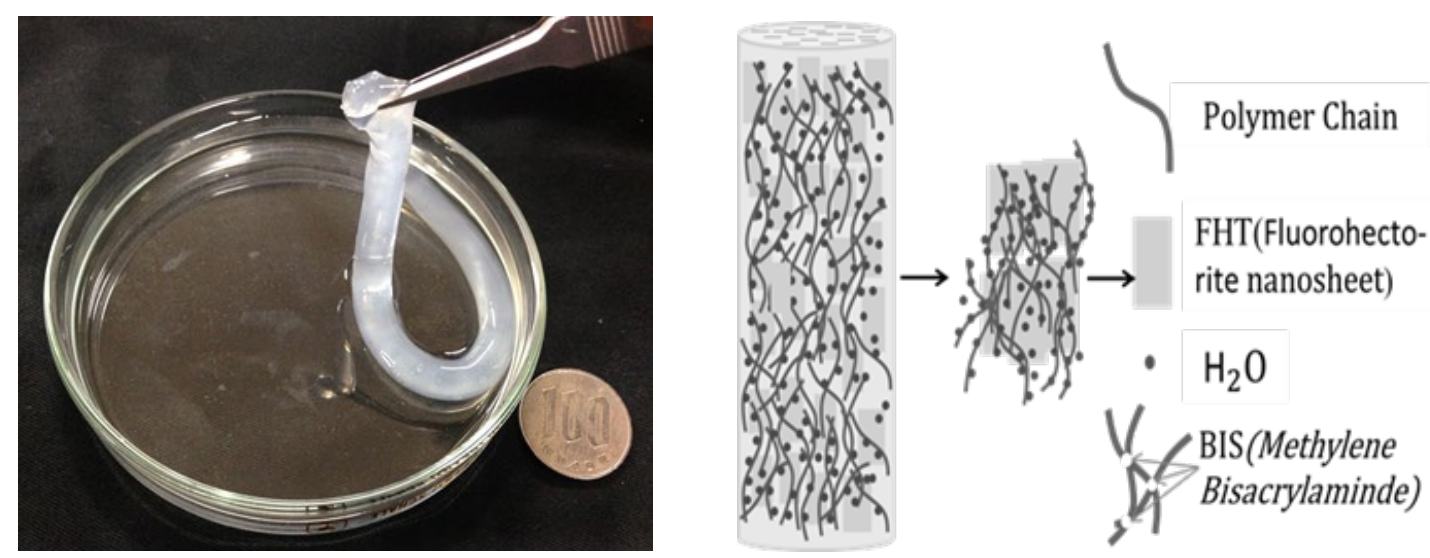

Figure 1: Photograph and composition of the soft gel actuator (Polymer Chain $6.8 \%$, FHT nanosheet $1.0 \%, \mathrm{H}_{2} \mathrm{O} 92.1 \%$, Methylene-bisacrylamide $0.1 \%$ ). 

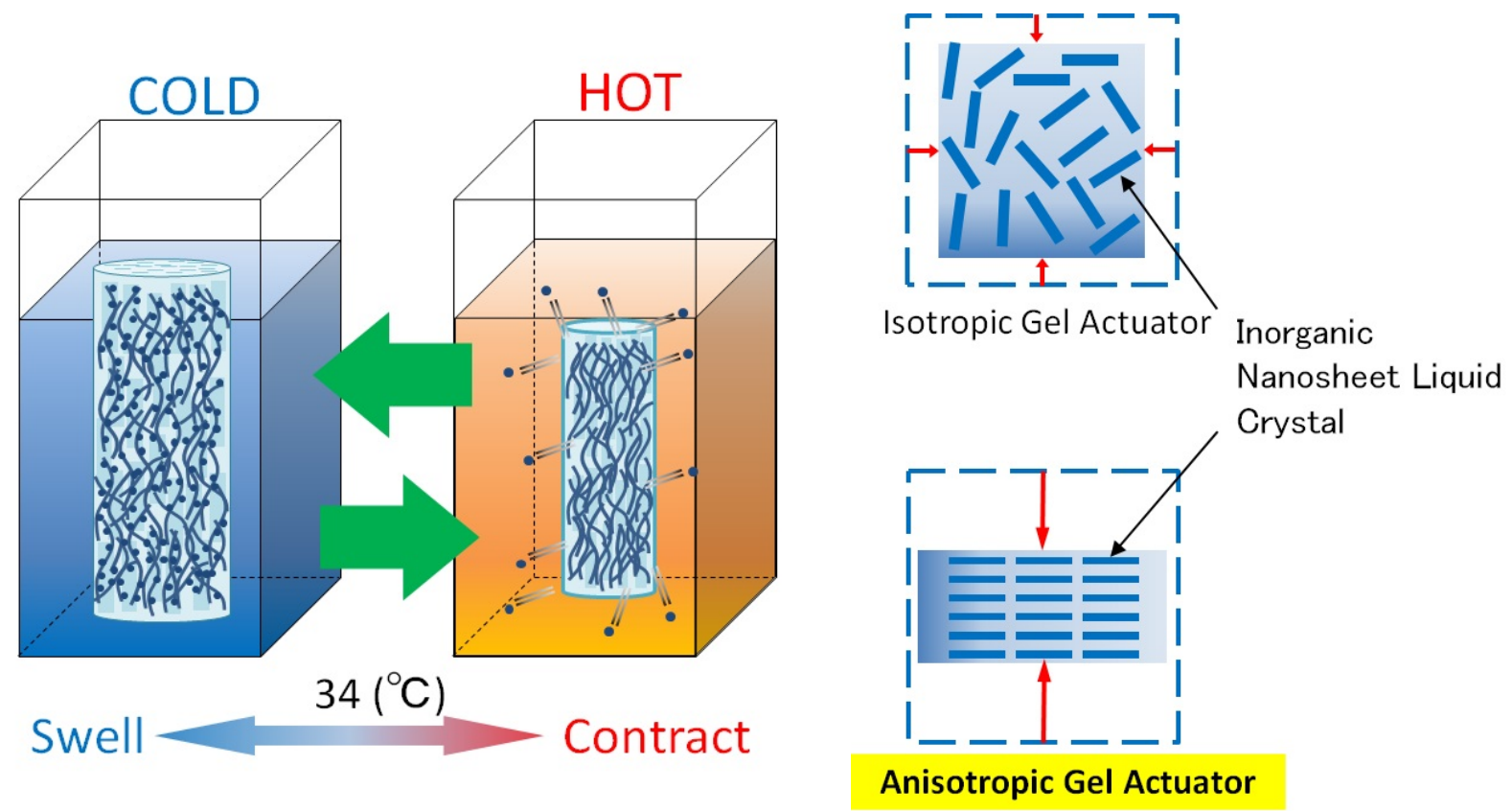

Figure 2: Contraction mechanism and anisotropic contraction depending on nanosheet orientation.

As shown in Fig. 2, when the gel in water is heated to more than the transition temperature (approximately 34 [deg C]) [7], the water molecules inside the gel are moved outside; the gel contracts. Conversely, when it is cooled, the surrounding water molecules are absorbed into the gel; the gel swells.

Such thermal characteristics can be generally observed in other previous gel actuators that have the isotropic deformation. However, this gel actuator remarkably has an anisotropic contraction characteristic because the orientation of the nanosheets is uniformly arranged; for example, as shown in Fig. 2, the horizontal arrangement of the nanosheet orientation enables the contraction in the vertical direction to be larger than in the horizontal direction [8].

In the previous paper [9], the authors' research group experimentally clarified the static characteristic of thermal contraction for the gel actuator and demonstrated length control based on the static thermal characteristic. However, little is known about the dynamic characteristics of the thermal contraction for this gel actuator.

In this paper, as a basic analysis, the heating step response is investigated through experiments, and the variation in the time constant against the dimensions is revealed. The second section illustrates the experimental setup for the measurement of the heating step response; in the third section, the experimental results are demonstrated. The paper's results are summarized in the last section, which contains the conclusions.

\section{Experimental Setup}

To measure the heating step response, we built the experimental setup as shown in Fig. 3. The experimental setup consisted of a water tank, a stirrer, a digital camera, a heater, and a gel holder. The water tank had sufficient hot water that was heated by the heater and stirred by the stirrer to uniformly keep the water temperature at approximately 76 [deg C].

The gel holder played the role fixing a test piece of the gel actuator, which was allowed to freely fall into the hot water along guide rails quickly. The gel holder consisted of two sheets and a restraint device; in the right column of Fig. 3, parts (a) and (b) show the aspects when the sheets open and close. Each sheet had four square-opening-spaces in which the surrounding hot water could smoothly pass through into a test piece. The restrained device in the holder prevented a test piece from dynamically float-moving, but its thickness was larger than a test piece in such a way that there were gaps between the piece and the sheets. In the experiment, after the free-fall of the holder, the time variation of the test piece contraction was measured by the use of an external digital camera. 


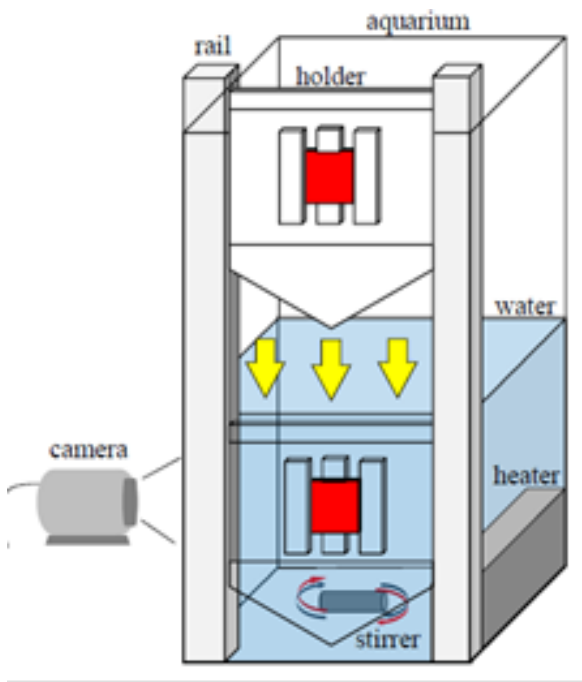



(a)

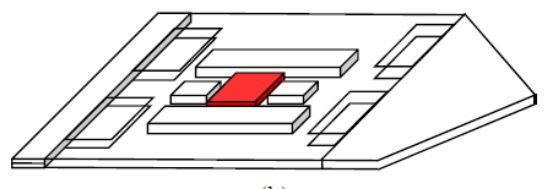

(b)

Figure 3: Experimental setup and gel holder.

For this experiment, we prepared six types of feature sizes (A-F) for the test pieces of the gel actuator, as shown in Table 1. The test pieces were thin squares and had anisotropic contraction; the orientation of the nanosheets was uniformly arranged by the use of an electric-field. By the uniform arrangement of the nanosheets, the contraction was larger in the vertical direction.

The shape of a test piece, the vertical length and horizontal length, were measured with the use of OpenCV through camera-shoot data; however, the thickness was not measured. The test pieces were red-colored for the image processing of OpenCV. When accounting for the individual differences, three pieces were prepared for each feature size. Then, three measurements of the heating step response were made for each test piece; therefore, nine measurements were made for each size.

To measure the heating step response, first, a test peace at room temperature (approximately 20 [deg $\mathrm{C}]$ ) was set in the holder as the large contraction pointed to the vertical direction, and then, the holder with a test piece was free-dropped from the initial position. In this experiment, when the holder reached the bottom of the water tank, the measurement started when the time was zero $(t=0[\mathrm{~s}])$; the total measurement time was 600 [s]. The falling gel took a brief period to reach the bottom from contacting the surface of the water over the tank; the test piece was contracted even while it was falling into the water. However, it is assumed that the contraction while falling into the water is ignorable because the time constant of the test pieces is sufficiently high.

Table 1: Feature sizes of the test pieces (A-F).

\begin{tabular}{|c|c|c|}
\hline Test pieces & Size $[\mathrm{mm}]$ & Thickness [mm] \\
\hline A & $10.0 \times 10.0$ & \multirow{2}{*}{1.0} \\
\hline B & $5.0 \times 5.0$ & \\
\hline C & $2.0 \times 2.0$ & \multirow{2}{*}{0.5} \\
\hline D & $10.0 \times 10.0$ & \\
\hline E & $5.0 \times 5.0$ & \\
\hline F & $2.0 \times 2.0$ & \\
\hline
\end{tabular}




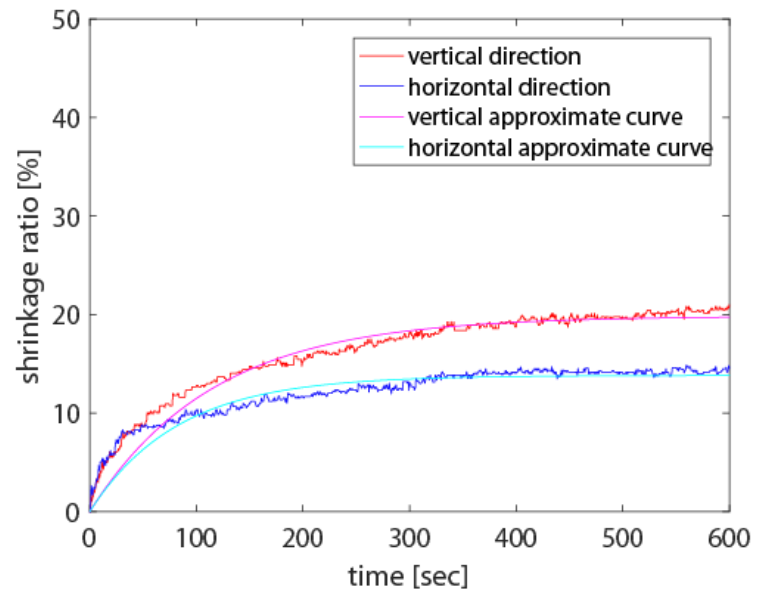

(a) Test pieces $\mathrm{A}$

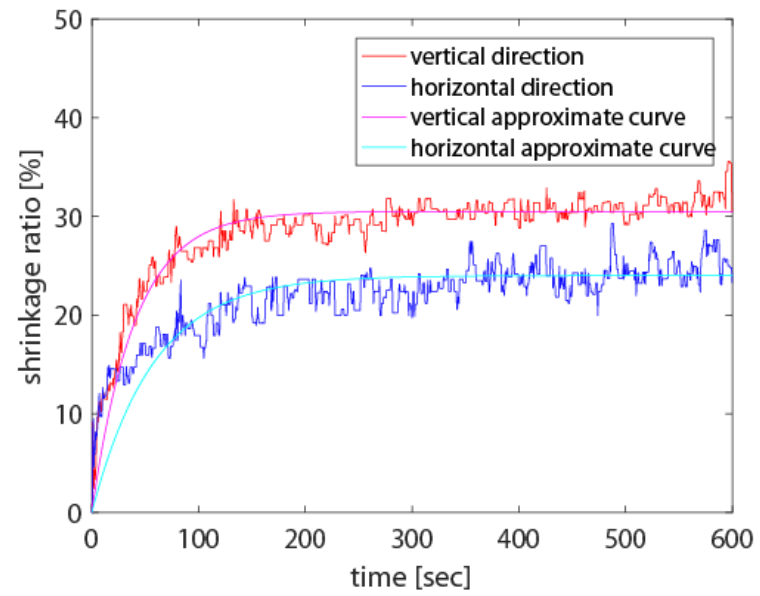

(b) Test pieces F

Figure 4: Examples of experimental results (heating step response of $\mathrm{A}$ and $\mathrm{F}$ ).

\section{Experimental Results}

The step response of sizes A and F are shown in Fig. 4 as typical examples. The vertical scale shows the contraction ratio; the red lines mean the average variation of three pieces in the vertical direction; and the blue lines mean the variation in the horizontal direction.

As a result, it is found that the vertical contraction is larger than the horizontal direction, and that the step response behaves similar to a first-order lag element. Therefore, in this paper, the gel actuator is assumed to be a first-order lag system, described as in Eq. (1), although the dynamic model of the gel actuator has not been clear in detail up to this point.

$$
\mathrm{y}(\mathrm{t})=K\left(1-e^{-\frac{t}{T}}\right)
$$

where $\mathrm{y}(\mathrm{t})$ is the resultant contraction ratio; $K$ is the gain constant; and $T$ is the time constant. The least-squares-approximated curves using Eq. (1) are drawn as blue and red narrow lines in Fig. 4.

In this paper, the response characteristic of the gel actuator is evaluated by use of the values of the time constant $T$. Fig. 5 demonstrates the variations in the time constant for the test pieces A-F; Fig. 5(a) is for test pieces A, B, and C, of which had the thickness of 1 [mm], and Fig. 5(b) is for D, E, and $\mathrm{F}$, which had the thickness of 0.5 [mm].

In the figure, the red lines mean the vertical time constant, and the blue lines mean the horizontal times constant. From Fig. 5, it is apparent that the time constant in the vertical direction decreases with a decrease in the planar dimension; thus a smaller planar dimension makes the constriction faster. However, the relative nature of their specific surface areas is not clearly observed in this experiment.

\section{Conclusions}

This paper investigated the heating step response of a soft gel actuator that is hybridized with nanosheet liquid crystals through experiments. The experimental results showed that the gel actuator can be regarded as a first-order lag system and demonstrated that the value of the time constant tends to be smaller when the planar dimension is smaller; specifically, a smaller planar dimension makes the constriction faster. However, the relative nature of their specific surface areas was not clearly observed. 

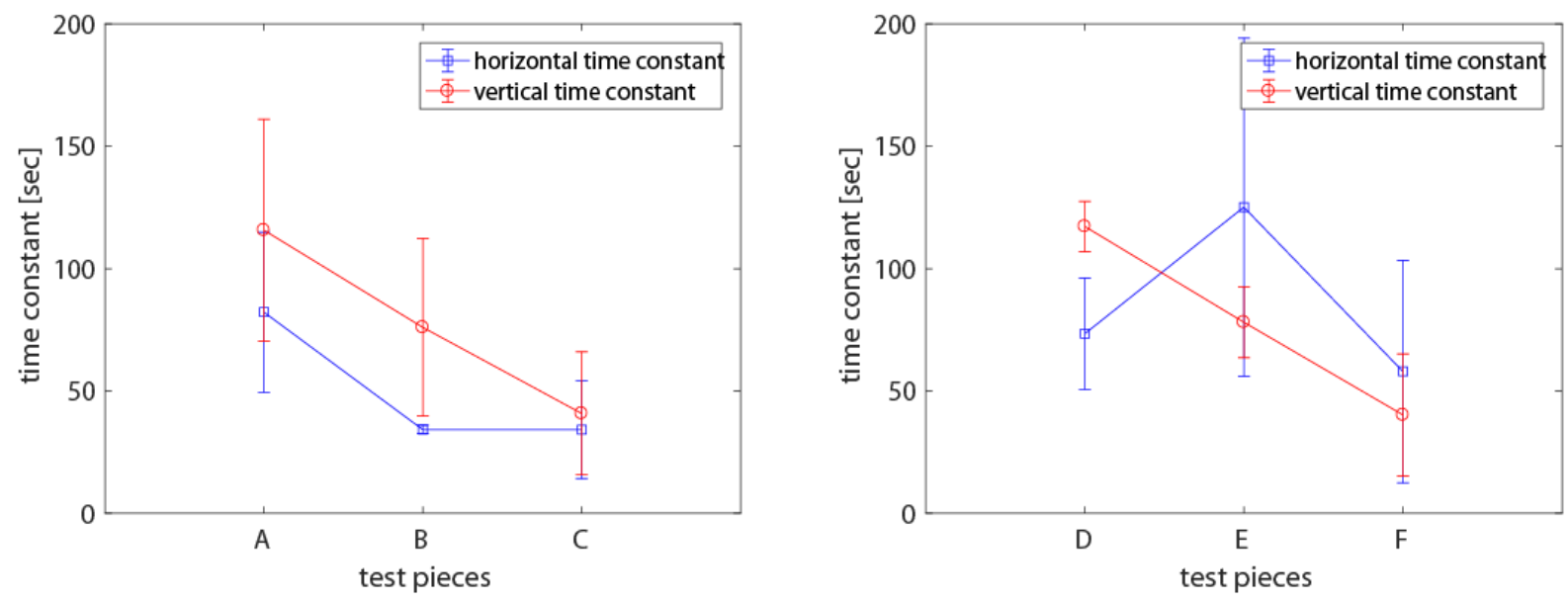

Figure 5: Variations in the time constant depending on the size.

In this paper, the step response was investigated only for heating; however, this property is different between heating and cooling. Therefore, the investigation of the cooling step response is also very important. Furthermore, the clarification of the frequency characteristics with heating and cooling will be the subject of a future study.

\section{References}

[1] Y. Osada, H. Ozaki, H. Hori: A Polymer Gel with Electrically Driven Motility, Nature, 355, pp. 242-244 (1992).

[2] Y. Okumura, K. Ito: The Polyrotaxane Gel: A Topological Gel by Figure-of-Eight Cross-link, Adv. Mater., 13, pp. 485-487 (2001).

[3] K. Haraguchi, T. Takeshisa: Nanocomposite Hydrogels: A Unique Organic Inorganic Network Structure with Extraordinary Mechanical, Optical, and Swelling/De-swelling Properties, Adv. Mater., 14, pp. 1120-1124 (2002).

[4] R. Urayama, S. Honda, T. Takigawa: Electrooptical Effects with Anisotropic Deformation in Nematic Gels, Macromolecules, 38, pp. 3574-3576 (2005).

[5] S. Maeda, Y. Hara, H. Sakaki, R. Yoshida, H. Hashimoto: Self-Walking Gel, Adv. Mater., 19, pp. 3480-3484 (2007).

[6] S. M. Douglas, I. Bachelet and G. M. Church: A Logic-Gated Nanorobot for Targeted Transport of Molecular Payloads, Science, 335, pp. 831-834 (2012).

[7] Y. Tanaka: Collapse of Gels and the Critical Endpoint, Phys. Rev. Lett., 40, pp. 820-823 (1978).

[8] N. Miyamoto, M. Shintate, S. Ikeda, Y. Hoshida, Y. Yamauchi, R. Motokawa and N. Annaka: Liquid Crystalline Inorganic Nanosheets for Facile Synthesis of Polymer Hydrogels with Anisotropies in Structure, Swelling/Deswelling, and Ion Transport/Fixation, Chem. Commun., 49, pp. 1082-1084 (2013).

[9] H. Kino, N. Samrejfuangfoo, K. Tsuda, T. Kato, H. Fujioka, N. Miyamoto: Basic Study of Soft Actuator Using Anisotropic Gel Hybridized with Nanosheet Liquid Crystal: Analysis of heat characteristics and length control, J. Procedia Computer Science, 105, pp 62-67 (2017). 